\title{
A scheme for generation of multi-photon GHZ states with cross-Kerr nonlinearities
}

\author{
Ting-Ting $\mathrm{Xu}^{*}$, Wei Xiong, and Liu Ye \\ School of Physics and Material Science, Anhui University, Hefei 230039, China
}

Received 1 March 2011; Accepted (in revised version) 22 March 2011

Published Online 8 December 2012

\begin{abstract}
With the help of cross-Kerr nonlinearities and homodyne measurement, we propose a scheme to generate three-photon GHZ states. Under the same framework, multi-photon GHZ state can be also realized. Compared with the previous ones, our scheme is much more simplified and lower errors. Moreover, only some linear elements and weak cross-Kerr mediums are employed, which make our scheme more feasible in the experiment.
\end{abstract}

PACS: 03.67.-a

Key words: GHZ state, cross-Kerr nonlinearity, quantum entanglement

\section{Introduction}

Entanglement is an important resource of quantum computation and quantum information processing (QIP) [1-6]. It is one of the most important ingredients of various intriguing phenomena [7-9]. The property of entanglement has been investigated both in bipartite systems [10,11] and multipartite systems[12,13]. At present, multipartite entanglement has attracted increasing interest because of its superiority during QIP. For example, the Greenberger-Horne-Zeilinger (GHZ) state is an important class of multipartite entangled states. GHZ state maximally violates Bell-type inequalities; the mutual information of measurement outcomes is maximal; it is maximally stable against (white) noise, and one can locally obtain an EPR state shared between any two of the three particles from a GHZ state with unit probability. Additionally, GHZ state can be employed as a quantum channel for quantum key distribution [14] and quantum secret sharing [15] and so on. In recent years, there has been much progress on the experimental generation of highly entangled states. Five-photon entanglement has been observed and used to

${ }^{*}$ Corresponding author. Email address: xtt1110@163.com (T. T. Xu) 
realize open-destination teleportation [16]. Six-particle GHZ states and eight-particle W states have been demonstrated in ion traps [17, 18].

Consequently, preparation of GHZ states have been attracted by many researchers. For example, Huang et al. have generated a three-qubit GHZ state by only one-step quantum operation [12]. Yang [19] provided a preparation of N-qubit GHZ entangled states in cavity QED, and Sharma et al. [20] have generated GHZ state in a quantum dot molecule, and so on.

Recently, as possibility of long-distance transmission with relatively low decoherence and quite simple manipulation of the states with linear optics, much attention has been paid to optical cross-Kerr medium, and this cross-Kerr effect involves two optical field modes, one is called signal mode and the other probe mode. A weak interaction between photons in these two modes is induced by passing them through nonlinear Kerr media. Cross-Kerr nonlinearity provides a good tool to construct the quantum non-demolition detection (QND), which has the potential of being able to condition the evolution of our system without the necessity of destroying the single photons [21-23], and which, with a cross-Kerr medium and a coherent state, can be used for checking the parity of the polarizations of two photons, operating as a controlled-Not (CNOT) gate [21], and analyzing the Bell states [24]. Recently, cross-Kerr nonlinearity has been used for many researches, for example, the generation of cluster state [25,26] and Dicke states [27].

The Hamiltonian of cross-Kerr nonlinear media can be described by $H_{Q N D}=\hbar \chi \hat{n}_{p} \hat{n}_{s}$. The symbol $\hat{n}_{p}\left(\hat{n}_{s}\right)$ denotes the number operator for the mode probe (signal), and $\hbar \chi$ is the coupling strength of the nonlinearity, which is determined by the property of the material used. Initially, if we consider the signal state have the form $|\varphi\rangle_{s}=a|0\rangle_{s}+b|1\rangle_{s}$, the probe beam is in a coherent state $|\alpha\rangle_{p}$, then the cross-Kerr interaction causes the combined signal-probe system to evolve as

$$
\psi(t)_{\text {out }}=U_{c k}|\varphi\rangle_{s}|\alpha\rangle_{p}=e^{\left(i H_{Q N D} t\right) / n}\left(a|0\rangle_{s}+b|1\rangle_{s}\right)|\alpha\rangle_{p}=a|0\rangle_{s}|\alpha\rangle_{p}+b|1\rangle_{s}\left|\alpha e^{i \theta}\right\rangle_{p}
$$

where $\theta=\chi t$ and $t$ is the interaction time. We observe immediately that the Fock state $|n\rangle_{s}$ is unaffected by the interaction with the cross-Kerr nonlinearity but the coherent state picks up a phase shift directly proportional to the number of photons $n_{s}$ in the signal $|n\rangle_{s}$ state. If we could measure this phase shift we could then infer the number of photons in the signal mode. This can be achieved simply with a homodyne measurement and conditional on the results of the homodyne measurement [24]. In our scheme, we employ the model proposed by Guo et al. [25](as shown in Fig. 1) to realize three-photon GHZ state.

Obviously, our scheme also possesses the advantages as well as theirs, and our scheme can be easily scaled to multi-photon GHZ state, which may be useful for quantum computation and quantum communication.

The paper is arranged as follows: In Section 2, the model is introduced and a scheme is proposed for the generation of the GHZ state of three photons. In Section 3, the scheme in Section 2 is scaled to N-photon GHZ state. The conclusion and the discussion will be shown in Section 4. 


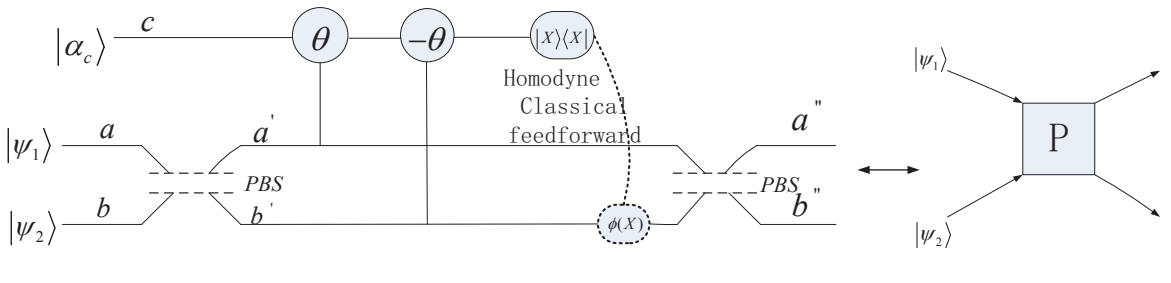

(a)

(b)

Figure 1: (a). The parity meter for photonic qubit coding in the polarization degree of freedom. PBS indicates polarizing beam splitter. The $\theta$ indicates that the coherent beam in mode $C$ will pick up a phase shift $\theta$ when there is a photon in mode $\alpha . \phi(X)$ represents a phase shift dependent on the feed forward result of the homodyne measurement. (b). The circuit shown to the Fig. 1(a).

\section{Scheme for generation of GHZ states of three photons}

Based on the cross-Kerr nonlinearities and the homodyne measurement, we proceed with our scheme. As shown in Fig. 1, it consists of two polarization beam splitters (PBS), the probe beam is the coherent state $\left|\alpha_{c}\right\rangle$, and two polarization photons are in the form $\left|\psi_{1}\right\rangle=\alpha|H\rangle+\beta|V\rangle,\left|\psi_{2}\right\rangle=\delta|H\rangle+\gamma|V\rangle$, where $|\alpha|^{2}+|\beta|^{2}=1$, and $|\delta|^{2}+|\gamma|^{2}=1$.

After the first PBS, the two photons will be presented in the mode $\mathrm{a}^{\prime}$ and $\mathrm{b}^{\prime}$ separately, and through the cross-Kerr interaction, the photon in mode a' will pick up phase shift $\theta$ and another photon in mode $b^{\prime}$ will pick up $\theta^{\prime}$, and the subsequent PBS is used to separate two photons into different spatial modes, and then the joint state evolves into

$$
\left|\psi_{12 \alpha c}\right\rangle=(\alpha \delta|H H\rangle+\beta \gamma|V V\rangle)\left|\alpha_{c}\right\rangle+\left(\alpha \gamma|H V\rangle\left|\alpha_{c} e^{-i 2 \theta}\right\rangle+\beta \delta|V H\rangle\left|\alpha_{c} e^{i 2 \theta}\right\rangle\right)
$$

Then we can obtain the projection of $\left|\psi_{12 \alpha_{c}}\right\rangle$ onto the eigenstate $|x\rangle$ of the observable $\mathrm{X}$ depending on $\mathrm{X}$ homodyne measurement. After the measurement, the two states we obtain are $\alpha \delta|H H\rangle+\beta \gamma|V V\rangle$ and $e^{-i \phi(x)} \alpha \gamma|H V\rangle+e^{i \phi(x)} \beta \delta|V H\rangle$. If the result of homodyne detector is $\left|\alpha_{c} e^{-i 2 \theta}\right\rangle\left(\left|\alpha_{c} e^{i 2 \theta}\right\rangle\right)$, the state of these two photons is collapsed into $|H V\rangle(|V H\rangle)$. In order to gain the EPR state deterministically, we choose a classical feed forward from the probe phase (the dashed line in Fig. 1 can be performed to transform this state to $\alpha \gamma|H V\rangle+\beta \delta|V H\rangle)$, then the state $\left|\alpha_{c} e^{ \pm i 2 \theta}\right\rangle$ cannot be distinguished [24].

Assume two polarization photons with the form $|+\rangle_{1}$ and $|+\rangle_{2}$, where $|+\rangle_{i}=\frac{1}{\sqrt{2}}(|H\rangle+$ $|V\rangle),(i=1,2)$, and $\left|\psi_{12}\right\rangle=|+\rangle_{1} \otimes|+\rangle_{2}$. Under the same situation, we let the system write into a P system(Fig. 1(b)). Additionally, when the result we obtain is $|H H\rangle+|V V\rangle$, we let $P=0$; and when the result is $|H H\rangle+|V V\rangle$, we let $P=1$. When $P=0$, the initial state will collapse to $\left|\psi_{12}^{0}\right\rangle=\frac{1}{\sqrt{2}}(|H H\rangle+|V V\rangle)$, and we choose $\sigma=I$; and when $P=1$, we can obtain the same state by performing $\sigma=\sigma_{x}$ on the second photon, here $\sigma_{x}$ is Pauli X operator. It is evident that Fig. 2 is an encoder for Bell state.

Now, as shown in Fig. 2, we let three initial states $|+\rangle_{i}(i=1,2,3),\left|\psi_{123}\right\rangle=|+\rangle_{1} \otimes$ $|+\rangle_{2} \otimes|+\rangle_{3}$ pass through the PBSs and cross-Kerr nonlinearities, when $P_{1}=0$, the initial 


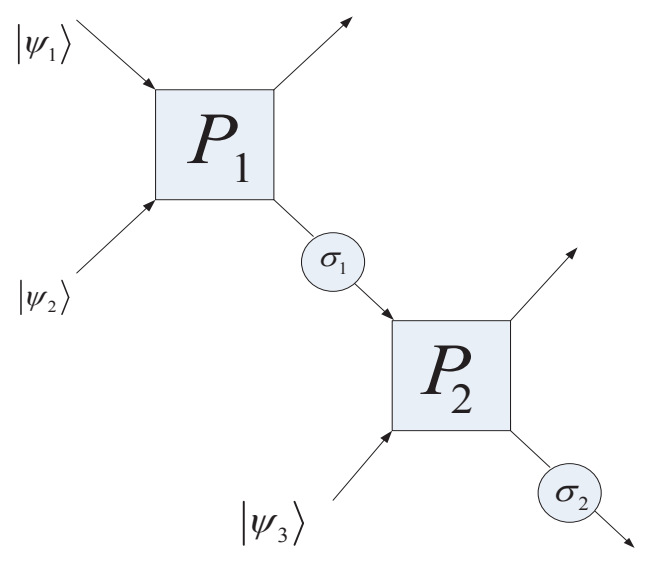

Figure 2: The setup for generating three-photon GHZ state.

state will be evolved into the state

$$
\left|\psi_{123}\right\rangle=\frac{1}{\sqrt{2^{3}}}\left[(|H H H\rangle+|V V V\rangle) \alpha+\left(|H H V\rangle \alpha e^{-i 2 \theta}+|V V H\rangle \alpha e^{i 2 \theta}\right)\right]
$$

when $P_{2}=0$, we perform operations $\sigma_{1}=\sigma_{2}=I$ on photons 2 and 3 , then the state of Eq. (3) will be changed into three-photon GHZ state $\left|\psi_{\text {out }}\right\rangle=\frac{1}{\sqrt{2}}(|H H H\rangle+|V V V\rangle)$; when $P_{2}=1$, the state of Eq. (3) will be collapsed into $\left|\psi_{\text {out }}^{\prime}\right\rangle=\frac{1}{\sqrt{2}}(|H H V\rangle+|V V H\rangle)$, respectively. Obviously, $\left|\psi_{\text {out }}^{\prime}\right\rangle$ can be transformed into the state $\left|\psi_{\text {out }}\right\rangle$ by performing operation $\sigma_{2}=\sigma_{x}$ on the third photon.

On the contrary, when $P_{1}=1$, the state of two photons can be evolved into the state $\left|\psi_{12}^{0}\right\rangle$ by performing operation $\sigma=\sigma_{x}$ on the second photon, then the initial state will be evolved into Eq. (3), so the following process can be performed as well as the condition $P_{1}=0$. Until now, the three-photon GHZ state can be generalized.

\section{Extension of the scheme}

In Section 2, we have obtained the GHZ state. Straightforwardly, an N-particle GHZ states can be generated easily using the $N$ encoders proposed above in series, as shown in Fig. 3.

And $\sigma_{i}=I$ for $P_{i}=0$, while $\sigma_{i}=\sigma_{x}$ for $P_{i}=1$. Now consider $\mathrm{N}$ photons prepared initially in a product state $\left|\psi_{0}\right\rangle=\otimes_{i=1}^{N}|+\rangle_{i}$, after $n-1$ successive encoder P and feed forward, the whole state becomes

$$
\begin{aligned}
\left|\psi^{\prime}\right\rangle= & \frac{1}{\sqrt{2^{n}}}\left[(|H H \cdots H\rangle+|V V \cdots V\rangle) \otimes\left|\alpha_{c}\right\rangle_{1} \cdots\left|\alpha_{c}\right\rangle_{n}+|H H \cdots V\rangle\left|\alpha_{c}\right\rangle_{1} \cdots\left|\alpha_{c} e^{-i 2 \theta}\right\rangle_{n}\right. \\
& \left.+|V V \cdots H\rangle\left|\alpha_{c}\right\rangle_{1} \cdots\left|\alpha_{c} e^{i 2 \theta}\right\rangle_{n}\right]
\end{aligned}
$$




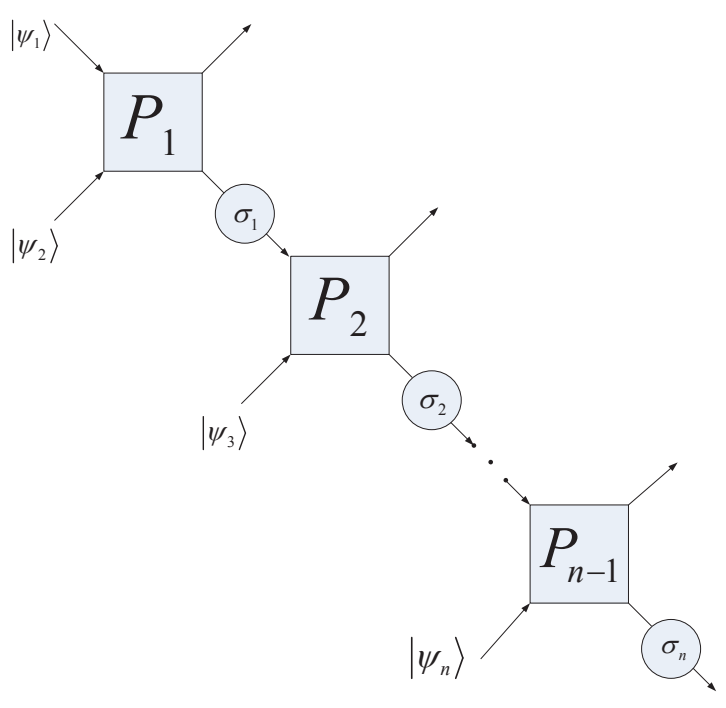

Figure 3: The setup for generating an N-particle GHZ state, which is $\mathrm{N}$ encoders in Fig. 2.

then we selecting appropriate $P_{i}$ and $\sigma_{i}$ as before.

When $P_{1}=P_{2}=\cdots=P_{n-2}=0, P_{n-1}=1$, we should let $\sigma_{1}=\sigma_{2}=\cdots=\sigma_{n-1}=I$, we can obtain $N$-photon GHZ state straight fully. Under other conditions, we can also obtain $\mathrm{N}$-photon GHZ state, the detailed analysis are shown as following:

(i) When $P_{1}=P_{2}=\cdots=P_{n-2}=0, P_{n-1}=1$, we can obtain the state

$$
|\phi\rangle_{i}=\frac{1}{\sqrt{2^{n}}}\left[\left|(H H \cdots H)_{n-1} V_{n}\right\rangle+\left|(V V \cdots V)_{n-1} H_{n}\right\rangle\right]
$$

Then we can perform operations $\sigma_{n-1}=\sigma_{x}$, and $\sigma_{1}=\sigma_{2}=\cdots=\sigma_{n-2}=I$, so we can obtain the state $\left.|\phi\rangle=\frac{1}{\sqrt{2}}(|(H H \cdots H\rangle+| V V \cdots V\rangle\right)_{n}$, which is N-photon GHZ state;

(ii) When $P_{1}=1, P_{2}=\cdots=P_{n-1}=0$, we can obtain the state $|\phi\rangle_{i i}=\frac{1}{\sqrt{2^{n}}}(|H V H \cdots H\rangle+$ $|V H V \cdots V\rangle)_{n}$. Then we can perform operations $\sigma_{1}=\sigma_{x}$, and $\sigma_{2}=\cdots=\sigma_{n-1}=I$ to obtain the state $|\phi\rangle$;

(iii) When $P_{1}=P_{2}=\cdots=P_{n-2}=1, P_{n-1}=0$, we can obtain the state $|\phi\rangle_{i i i}=\frac{1}{\sqrt{2^{n}}}(|H V \cdots V H\rangle+$ $|V H \cdots H V\rangle)_{n}$. Then we can perform operations $\sigma_{1}=\sigma_{2}=\cdots=\sigma_{n-2}=\sigma_{x}$, so we also can obtain the state $|\phi\rangle$;

(iv) When $P_{1}=P_{2}=\cdots=P_{n-2}=1$, we can obtain the state $|\phi\rangle_{i v}=\frac{1}{\sqrt{2^{n}}}\left[\left|(H V \cdots V)_{n-1} H_{n}\right\rangle+\right.$ $\left.\left|(V H \cdots H)_{n-1} V_{n}\right\rangle\right)$. Then we can perform operations $\sigma_{1}=\sigma_{2}=\cdots=\sigma_{n-1}=\sigma_{x}$, and we will obtain the state $|\phi\rangle$. 
After the above operations, we can acquire the $N$-qubit GHZ state

$$
|G H Z\rangle_{N}=\frac{1}{\sqrt{2}}(|H H \cdots H\rangle+|V V \cdots V\rangle)_{N} .
$$

\section{Conclusion}

Exploiting weak cross-Kerr nonlinearities, GHZ states are realized using current optical technologies. Our scheme only requires linear optical elements and homodyne detectors. The initial state consists of a single photon and coherent state. Through cross-Kerr interaction, the information of spatial modes can be transmitted to phase information of the coherent state. Now we consider the experimental realization of the present schemes. The main difficulty of the schemes with respect to an experimental demonstration consists in the requirement of single photon sources as input state. The complete technology of these resources is yet to be established [28, 29]. In summary, we have presented an effective scheme to generate multi-photon GHZ states with high success probabilities and near perfect fidelities. This approach takes advantage of the homodyne measurement which can be made much more efficient than the single-photon detection. Another important point with regard to the practical implementation of our scheme is that decoherence effects during the entanglement generation process in nonlinear media must be low. In additional, this scheme takes advantage of the nonlinear Kerr effect, and it is much more simplified and lower errors of the results. It uses only basic tools in quantum optical laboratories and can be implemented in the regime of the weak cross-Kerr nonlinearity. Our scheme is not limited to the all optical implication and can also be used in other QIP architectures with a cross-Kerr-like Hamiltonian [30].

Acknowledgments This work was supported by the National Science Foundation of China under Grant No. 11074002, the Doctoral Foundation of the Ministry of Education of China under Grant No. 20103401110003, and also by the Personal Development Foundation of Anhui Province under Grant No. 2008Z018.

\section{References}

[1] A. K. Ekert, Phys. Rev. Lett. 67 (1991) 661.

[2] C. H. Bennett, Phys. Rev. Lett. 70 (1993)1895.

[3] F. G. Deng and G. L. Long, Phys. Rev. A 70 (2004) 012311.

[4] N. Gisin and S. Massar, Phys. Rev.Lett. 79 (1997) 2153.

[5] A. Peres, Phys. Rev. Lett. 77 (1996) 1413.

[6] F. Girsldi and P. Grigolini, Phys. Rev. A 64 (2001) 032310.

[7] D. Boschi, S. Branca, F. De Martini, L. Hardy, and S. Popescu, Phys. Rev. Lett. 80 (1998) 1121.

[8] M. Hillery, V. Bužek, and A. Berthiaume, Phys. Rev. A 59 (1999)1829.

[9] M. A. Nielsen, Phys. Rev. Lett. 83 (1999) 436.

[10] A. Aspect, J. Dalibard, and G. Roger, Phys. Rev. Lett. 49 (1982) 1804.

[11] L. F. Wei, M. J. Storcz and F. Nori, Phys. Rev. A 73 (2006) 052307. 
[12] J. S. Huang, C. H. Oh, and L. F. Wei, Phys. Rev. A 83 (2011) 062108.

[13] J. Uffink, Phys. Rev. Lett. 88 (2002) 230406.

[14] J. Kempe, Phys. Rev. A 60 (1999) 910; R. Cleve, D. Gottesman, and H. K. Lo, Phys. Rev. Lett. 83 (1999) 648; V. Scarani and N. Gisin, Phys. Rev. Lett. 87 (2001) 117901; G. A. Durkin, C. Simon, and D. Bouwmeester, Phys. Rev. Lett. 88 (2002) 187902.

[15] M. Hillery, V. Buzek, and A. Berthiaume, Phys. Rev. A 59 (1999) 1829.

[16] Z. Zhao, Nature 430 (2004) 54.

[17] D. Leibfried, Nature 438 (2005) 639.

[18] H. Häffner, W. Hänsel, C. F. Roos, J. Benhelm, D. Chek-al-kar, M. Chwalla, T. Körber, U. D. Rapol, M. Riebe, P. O. Schmidt, C. Becher, O. Gühne, W. Dür, and R. Blatt, Nature 438 (2005) 643.

[19] C. P. Yang, Phys. Rev. A 83 (2011) 062302.

[20] A. Sharma and P. Hawrylak, Phys. Rev. B 83 (2011) 125311.

[21] K. Nemoto and W. J. Munro, Phys. Rev. Lett. 93 (2004) 250502.

[22] G. J. Miburn and D. F. Walls, Phys. Rev. A 30 (1984) 56.

[23] N. Imoto, H. A. Haus, and Y. Yamamoto, Phys. Rev. A 32 (1985) 2287.

[24] S. D. Barrett, P. Kok, K. Nemoto, R. G. Beausoleil, W. J. Munro1, and T. P. Spiller, Phys. Rev. A $71(2005)$ 060302(R).

[25] Q. Guo, J. Bai, L. Y. Cheng, X. Q. Shao, H. F. Wang, and S. Zhang, Phys, Rev. A 83 (2011) 054303.

[26] C. R. Zhao and L. Ye, Sci. China-Phys. Mech. \& Astron. 54 (2011 )497.

[27] C. R. Zhao and L. Ye, Phys. Lett. A 375 (2011) 401.

[28] C. Santori, M. Pelton, G. Solomon, Y. Dale, and Y. Yamamoto, Phys. Rev. Lett. 86 (2001) 1502.

[29] C. Brunel, B. Lounis, P. Tamarat, and M. Orrit, Phys. Rev. Lett. 83 (1999) 2722.

[30] P. van Loock, Phys. Rev. Lett. 96 (2006) 240501. 